\title{
Delay Sensitive and Interference Aware MAC Protocol for WSN
}

\author{
Srinivasan Gopalan ${ }^{1^{*}}$ \\ Murugappan Shanmugam $\mathbf{S}^{2}$ \\ ${ }^{1}$ Sathyabama University, Chennai, India \\ ${ }^{2}$ Annamalai University, Chennai Study Centre, Chennai, India \\ * Corresponding author’s Email: gsrinivasanphd@ gmail.com
}

\begin{abstract}
Interference is the most important issue in Wireless Sensor Network (WSN) as it causes severe degradation during communication. Also, as sensor nodes are power constraint, hence the system is expected to operate for longer duration without any delay during communication. In order to overcome these issue a delay sensitive and interference aware MAC protocol for WSN is proposed. In this paper, first MAC protocol is proposed which divides network into layer and assign node to each layer to avoid data loss in the network. Once network is divided to the multi-layer, timeslot is assigned to each node. To utilize the channel efficiently, timeslot steal mechanism is implemented. To avoid interference, a hybrid prioritization scheme is implemented to prioritize the packet queue based on weight of the packet queue and based on the idle time of the prioritized packet.
\end{abstract}

Keywords: Wireless Sensor Network; MAC protocol; hybrid prioritization scheme; Data packets; Delay.

\section{Introduction}

\subsection{WSN}

Wireless sensor networks (WSN) representing a new generation of embedded systems is a group of wireless mobile hosts forming nodes. The nodes arbitrarily and randomly alter their locations and communicate without the aid of any centralized administration or standard support services. Wireless Adhoc communication mechanisms are deployed for routing the sensory data from original sensor node to the control station. Nodes can be of sensor nodes or routers some nodes can be of both. A sensor node can be a data source in WSN by interacting with physical environment and collecting a specific data for control requirements e.g. temperature, humidity, pressure, movement, etc, producing sensory data. It can be a router to send data from a neighbor sensor node to other towards control station where the aggregates of collected data from various network sensors are processed and analyzed [1, 2]. WSNs are largely deployed in monitoring natural/wild life, production level machines in automation environments and earth movements, military, security, surveillance, health care, environmental monitoring, inventory tracking, industrial controls, agricultural and health-care applications etc. [3, 4].

WSNs have resource constrained individual devices with limited energy supplies, processing speed, storage capacity, and communication bandwidth and also power consumption [5]. Collision occurs when two nodes transmit at a same time and hence needed retransmission increasing power consumption. Overhearing and idle listening are also the issues, increasing the cost and power consumption [6].

\subsection{MAC protocol for delay sensitivity and interference awareness}

The medium access control (MAC) protocol has a significant role in finding the throughput, latency, bandwidth utilization and energy consumption of the network. Hence MAC protocol should be designed and chosen to provide the required QoS for a given application. Multi hop wireless sensor networks have several MAC protocols which can be topology dependent or independents. These protocols are can be divided into schedule and contention based MAC [4]. Also sensor MAC protocols can be classified as synchronous MAC protocols and asynchronous MAC protocols. Each node of synchronous MAC protocols simultaneously wakes up and sleeps. Whereas in 
asynchronous MAC protocols, each node asynchronously wakes up and check out the channel activity. Once the channel is busy, the radio is kept in the receiving state. Or else the node returns to the sleep state or transmits a sensing data packet [7].

Interference, the most significant issue in wireless network causes an irretrievable degradation of communications. Interference leads to data collisions and consequently data retransmissions. Hence a routing protocol should consider interference in path selection otherwise it leads to signal degradation etc. $[8,9,10]$.

The sensor node limitations like energy supply, computational and communication capabilities, common failures, etc., makes providing delay guarantee in WSN a hard task. In real time event driven applications, the end-to-end delay is the most critical factor since an event is to be reported to a sink as soon after its detection so as to take appropriate action immediately. Thus, an end-to-end delay guarantee is required by most applications for time sensitive data [11]. In previous works they have proposed a relay assisted effective loss recovery technique for WSN. In this technique, the relay nodes among the source and destination node are selected based on the parameters such as channel state information and combined score using a decentralized partially observable Markov decision process. The combined score for each sensor is defined involving the parameters such as queue length, link bandwidth, MAC contention and residual energy. Then the low density parity check (LDPC) codes are used to encode and decode for performing error recovery. As an extension to these works we propose to design a delay sensitive and interference aware MAC protocol to reduce the overall delay and interference among the nodes.

\section{Literature Review}

$\mathrm{N}$. Bandirmali et al [3] proposed an integrated approach of SEA together with the EDCMAC to provide a cost effective solution as well as to enable highly secure and energy-aware/delay sensitive data transfers in WSNs remaining functional much more long time.

S. A. Hameed et al [4] proposed a WSN MAC protocol (MD-SMAC) which is mobility-aware, delay-sensitive and provides satisfactory level of energy efficiency. However energy consumption is more.

S. Gherairi et al [5] proposed an optimized TDMA multi-frequency scheduling access contribution for sensor networks in three phases. The frequencies are assigned according to parent node reception in communication tree structure in the first phase. The number of frequencies was reduced according to graph coloring in second phase principle. Then the frequencies are reduced to the maximum number of allowed frequencies with TDMA (Time Division) clustering principle in third phase. However, the delay was increased.

B. M. Khan and F. H. Ali [6] proposed a novel MA-MAC protocol to provide a contention based collision free transmission utilizing back-off value allocation to the joining nodes rather than random selection of values to resolves control message collisions as well. The request mechanism was introduced before entering a new cluster to solve mobile node's association problem.

H. R. Oh and H. Song [7] proposed an energyaware wireless sensor MAC protocol for delaysensitive data transmission. A new preamble structure and a channel monitoring period selection algorithm was proposed for an effective tradeoff between energy consumption and delay with a low computational complexity since these two greatly depend on Channel Monitoring Period and data sensing period at each sensor node. However, estimation errors occur in overhearing, receiving and data sensing rate caused by a sudden change in traffic pattern or collision.

D. D. Chaudhary and Dr. L. M. Waghmare [12] proposed multi-sink model and designed probabilistic model to compare transmission delay in these two models. The innovative methodology was presented to deploy the sink nodes in WSN for the industrial control environment. The limitation of this protocol is that, its low throughput, because its packet drop ratio is large.

M. A. Yigitel et al [13] designed and implemented a QoS-aware MAC protocol for WMSNs, Diff-MAC, integrating various methods to satisfy QoS requirements to deliver heterogeneous traffic and a fair all-in-one QoS-aware MAC protocol was provided. The objective of Diff-MAC was to enhance the channel utilization with effective service differentiation mechanisms in addition to providing fair and fast delivery of the data. However, packet failures still occur due to buffer overflows.

M. A. Hamid et al [14] presented a scheduledbased multi-channel MAC protocol for enhancing network performance. Here each receiving node chose schedules/some timeslot(s), where it may receive data from the intending sender(s). The timeslot selection is done in such a way a node avoids the slots already chosen by others in its interference range. Also a unique solution was proposed dividing the neighboring nodes into different groups to reduce the conflicts during 
timeslot selection where nodes of a group may select the slots allocated to that group only.

M. Hossein, S. Gilani et al [15] proposed an adaptable CSMA/TDMA hybrid channel access method with some modifications in 802.15.4 standard. A part of contention access period was dedicated to a TDMA for energy and throughput. The drawback is higher end to end delay in long frames.

O. D. Incel et al [16] presented a multi-channel MAC protocol, MC-LMAC, designed to maximize WSN throughput by coordinating transmissions over multiple frequency channels. Interference and contention-free parallel transmissions were utilized on different channels. It relied on scheduled access to simplify node coordination of nodes, dynamical switching their interfaces between channels and makes the protocol operate effectively with no collisions during peak traffic. However there exist channel errors.

W. L. Tan et al [17] proposed an adaptive and energy-efficient TDMA-based MAC protocol to reduce network energy consumption in addition to efficiently handling network traffic load variations and optimizing channel utilization by a timeslot stealing mechanism and a timeslot reassignment procedure.

R. Thalore et al [18] presented an energyefficient multi-layer MAC (ML-MAC) protocol to achieve low duty cycle, prolonged network lifetime and minimum number of collisions. The energy consumption in communication is reduced by very short listening/active time of sensor nodes in MLMAC. Also, number of collisions is reduced saving the energy required to re-transmit corrupted data packets. However jitter and delay is increased.

\section{Problem Identification and Proposed Solution}

In existing works, they have proposed a reliable and energy efficient congestion control protocol in WSN. In this technique, energy efficient paths are established among source and sink that contains the nodes with sufficient energy levels. If the sink finds that the reliability index of the data transmission path is below the threshold, it chooses the alternate path with good reliability index. For detecting congestion in node level as well as network level, condition based on queue length, link bandwidth and MAC contention are executed. If congestion is detected, an efficient rate control mechanism is employed that adaptively alters the data transmission rate.

\subsection{Overview}

A MAC protocol dividing the nodes into layers are proposed where each node is assigned to these layers and data loss can be prevented [18]. Here the layer participating in communication is active and the rest are completely in sleep mode. This technique reduces power consumption as well as ensures reliable transmission reducing collisions since number of nodes transmitting the network is reduced.

Following, the number of processed time is set for each time and packets are classified according to time [13]. Then active time of node is set for refraining from both idle waiting time and due to sleeping next hops and unnecessary wastage of energy due to idle listening. A two-level hybrid prioritization scheme is implemented with one as traffic class type and next is the traversed hop count among the packets of the same traffic class. Different priority packet queues were maintained for each traffic class and a fair packet scheduler is integrated to the MAC protocol to choose the next serviced queue according to classified traffic requirements in multi-queue systems. Here fairness is provided among different priority traffic by efficient scheduling algorithms.

The timeslot estimated from above may be unused sometime causing wastage of energy. Hence we use a mechanism to enable unused timeslot to be used by another node so as to increase channel utilization [17]. In addition to primary sender node in which time slots are assigned, we assign a secondary sender node to each time slot which steals the timeslot once the timeslot is not used by primary node. For this purpose, secondary node must have to listen to channel to find whether primary node is transmitting. After timeout, it steals the time slot. This increase channel utilization and reduce average packet latency. Fig 1: represents the proposed block diagram. 


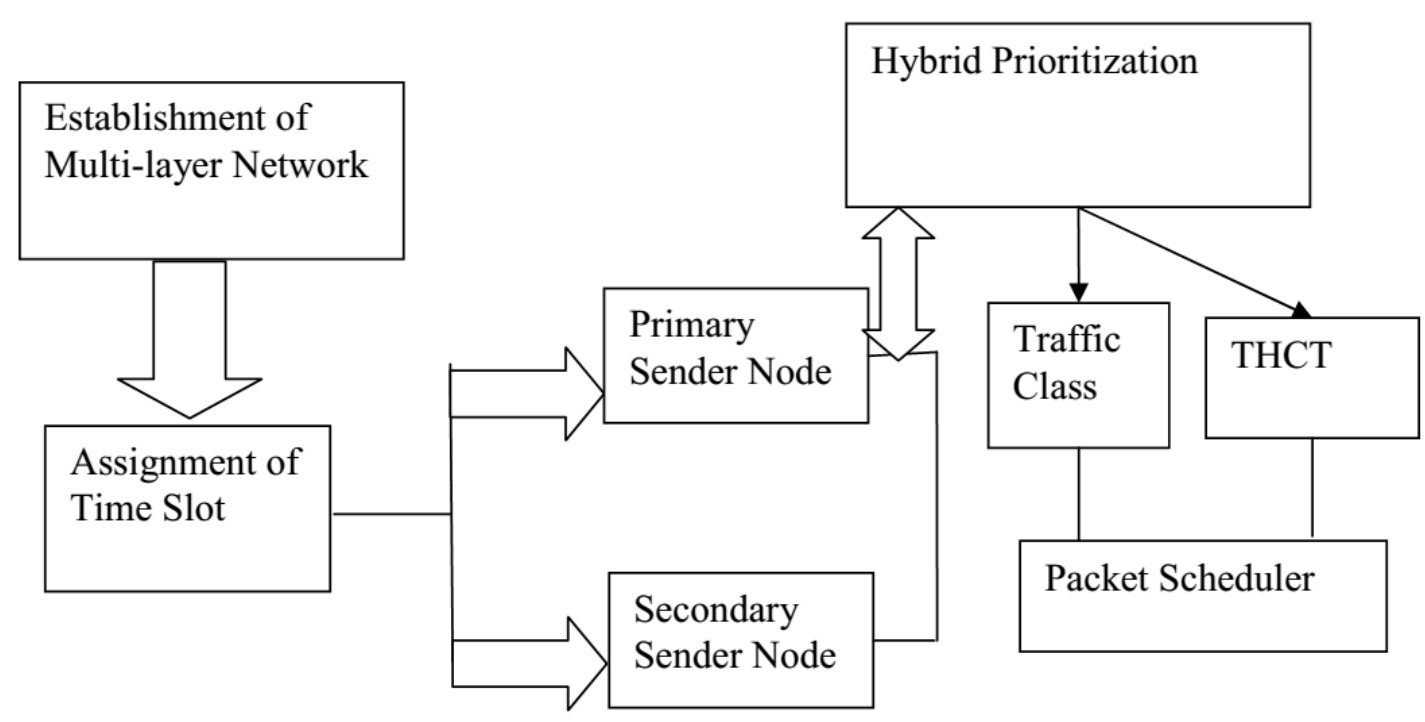

Figure.1 Block Diagram

\subsection{Multi-layer Network}

This section describes about the design of multilayer network [18].

Let $\left\{A_{1}, A_{2} \ldots A_{A}\right\} \in A$ represents set of nodes in the network which are further divided into $M$ layer such that the nodes in each layer cover the entire geographical area in which they are deployed. $\delta_{l}$, $\delta_{2} \ldots . . \delta_{M}$ represents the allotted time durations for network layers by considering the condition $\delta_{1}<$ $\delta_{2}<\ldots . .<\delta_{M}$

The total simulation time is the sum of active duration for all layers that which is given in equation (1):

$$
T_{O A}=T_{O A\left(M_{1}\right)}+T_{O A\left(M_{2}\right)}+\ldots . . . T_{O A\left(M_{M}\right)}
$$

At the time of simulation, first (A/M) nodes are active for the time period $T_{O A\left(M_{1}\right)}$ and is given as below:

$$
T_{O A\left(M_{1}\right)}=\left(\delta_{2}-0\right)
$$

Where the network starts at $0^{\text {th }}$ second.

Next $(A / M)$ nodes, i.e. $((A / M)+1)$ th node to $(2 A / M)$ th node are active for $T_{O A\left(M_{2}\right)}$ duration which is given as below:

Where $\delta_{1}<\delta_{2}$

$$
T_{O A\left(M_{2}\right)}=\left(\delta_{2}-\delta_{1}\right)
$$

Likewise last $(A / L)$ nodes, i.e. $((M-1) \times A / M)+1)$ th node to $A_{t h}$ are active for $T_{O A\left(M_{M}\right)}$ duration given as below:

$$
T_{O A\left(M_{M}\right)}=\left(\delta_{M}-\delta_{M-1}\right)
$$

Where $\delta_{L-I}<\delta_{L}$

\subsection{Assignment of Timeslot}

This section describes about the assignment of timeslot [17] to set of nodes in the multi-layer network. In order to increase the channel utilization, timeslots are assigned to each and every set of the node. To assign timeslot, nodes set in the multilayer network are divided into following two types:

$>$ Primary Sender Node: The active node to which the timeslot is assigned.

$>$ Secondary Sender Node: The nodes in the queue which utilize timeslot for transmission, if primary sender node doesn't utilize timeslot.

In order to make efficient channel utilization, secondary sender node steals the free time of the primary sender node. In order to do so, first secondary node listens carefully to the channel to determine whether the primary sender node is transmitting or not. This process is called as Clear Channel Assessment (CCA). In case after time out period, the secondary sender node doesn't listen or sense any channel activity, then it steals that time period for the transmission.

\subsubsection{Multi-layer Networking with Timeslot Mechanism}

This section describes about the implementation of timeslot with set of nodes in multi-layer network. This can be explained in the following steps:

Step 1: Assignment of timeslot to primary sender node $(\mathrm{P})$ and a secondary sender node $(\mathrm{S})$.

Step 2: Secondary Sender Node listen to channel and check for the condition

if node $P$ utilize the channel then node $S$ remain silent else $S$ utilize the timeslot. 


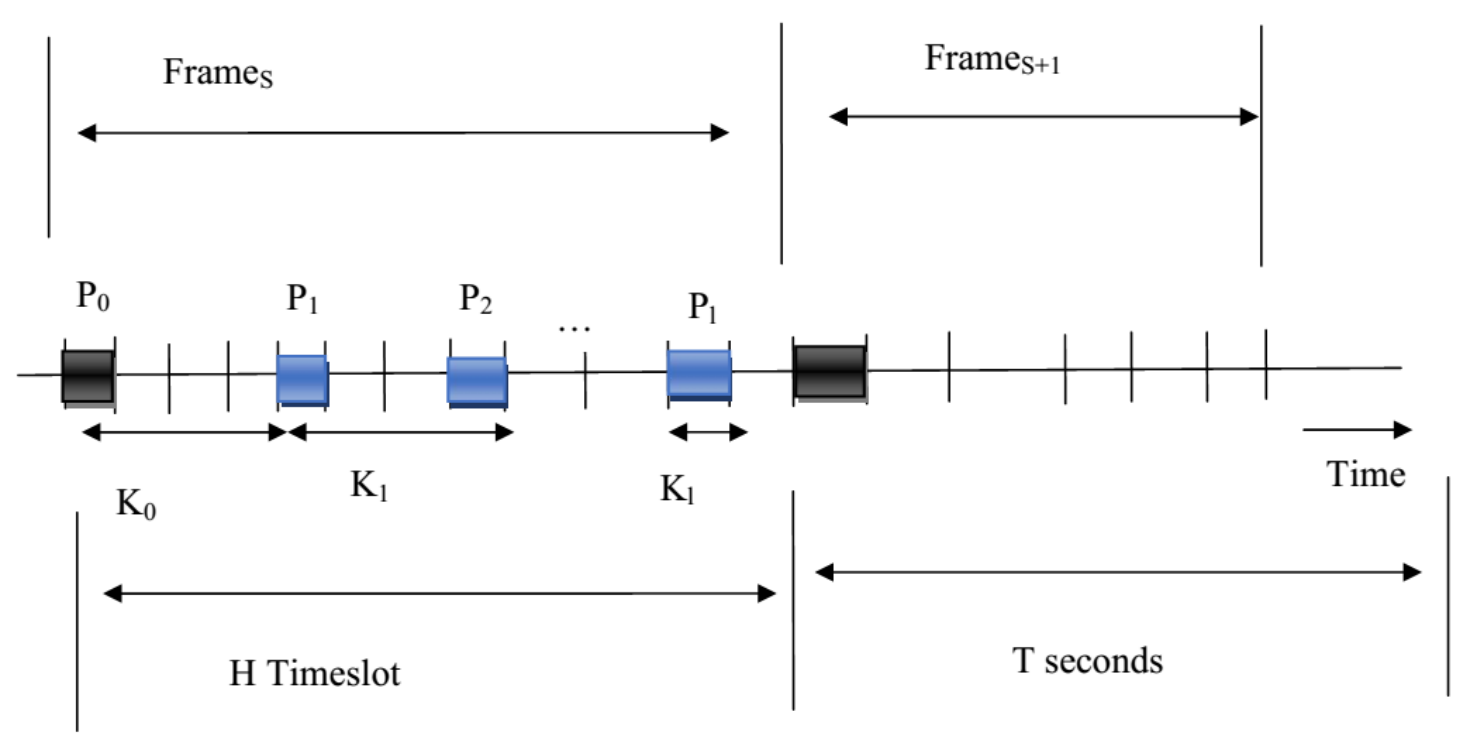

Figure.2 Timeslot assignment of Sender node

Hence, the number of timeslot in a frame that a sender node $P_{i}$ can utilize can be more than one. In Fig (2), example of assignment of the timeslot to transmit packet is shown. The black colored timeslot denotes the timeslots for which sender node $P_{i}$ is the primary sender node whereas red-colored timeslot denote the timeslot for which sender node $S_{i}$ represent the secondary sender node.

\subsubsection{Computation of Timeslot for Secondary Sender node}

In order to calculate average number of timeslot that a sender node $\mathrm{S}$ has in a time frame, the following terms are used [17],

$T_{F}$ - Frame duration

$H$-Number of timeslot in a frame

$\mathrm{N}$ - Number of sender Nodes contained in node set

$\mu_{i}-$ packet arrival rate at sender node $S$,

$S=1,2, \ldots . . n$

$G_{S^{-}}$Number of timeslot in a framework which is assigned to sender node $S_{i}$ as primary sender node.

$\sigma_{s}-$ utilization of sender node $S$

The use of sender node $S_{i}$ can be given by the following relationship:

$$
\begin{array}{r}
\sigma_{S}=\frac{\text { Numberof packet arrival in a frame }}{\text { Numberof Packet served in a frame }} \\
\sigma_{S}=\frac{\mu_{S} \boldsymbol{T}_{\boldsymbol{F}}}{\boldsymbol{G}_{S}}
\end{array}
$$

Where $G_{i}$ represents average number of timeslot that sender node $S$ has in a time frame $T_{F}$.

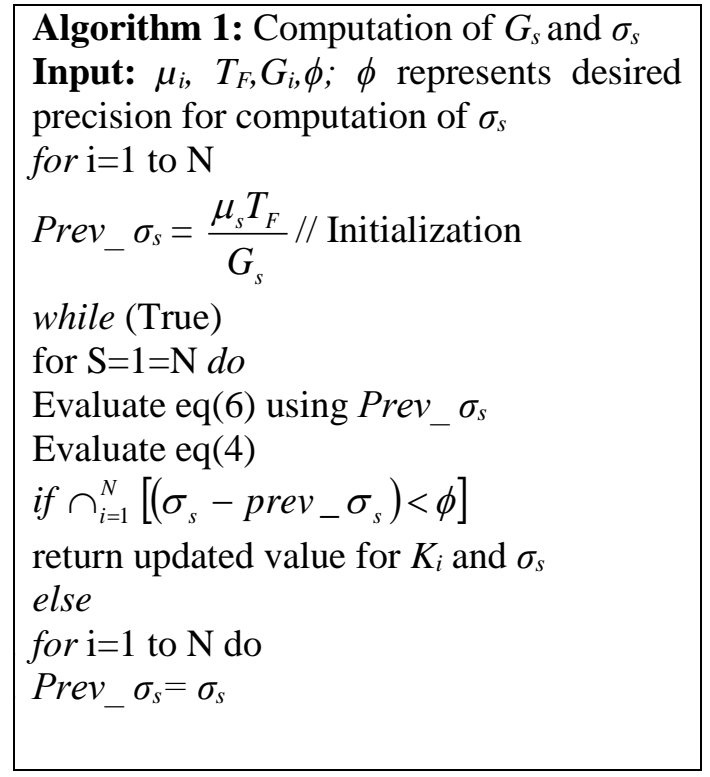

$G_{S}$ can be calculated as below:

$$
G_{S}=G_{S}+\sum_{P=1 ; P \neq S}^{N}\left(1-\sigma_{P}\right) G_{P ; S}
$$

Where $G_{P ; S}$ represents the number of timeslot in which sender node $P$ represents primary sender node and sender node $S$ represents secondary sender node. In this case compute, $G_{S}$ and Using Algorithm 1 which uses $G_{S}$ as the initial value for $G_{i}$ in its calculation.

\subsection{Hybrid Prioritization Technique}

Multi-layer Prioritized architecture is shown in fig 3. 


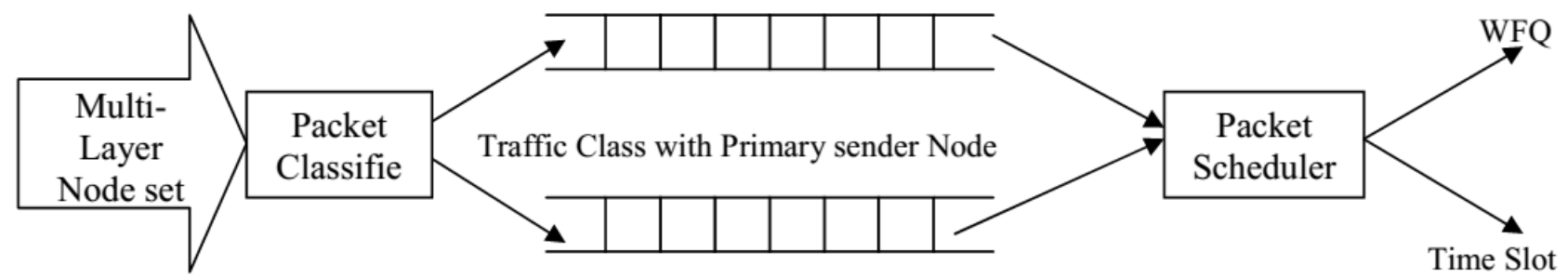

Traffic Class with Secondary sender Node

Figure.3 Multi-layer Prioritized Architecture

A two level hybrid prioritization technique [13] is implemented with first one as traffic class type and the second one as traversed hop count technique (THCT) amongst packet of the same traffic class. To maintain the priority of the packet queues for each traffic queues an efficient scheduling technique based on the Weighted Fair Queuing method is proposed. In this technique the packet scheduler select the next packet based on weight of the queue. The advantage of using WFQ is that medium sharing rates amongst traffic classes can be easily adjusted by altering the corresponding weight. This creates the ability to control the medium access and throughput for each traffic class. The traversed hop count is mainly based on energy, bandwidth, memory and active time allocated to them.

The following steps are involved to prioritize the packet queue based on the traffic class and traversed hop count technique:

Step 1: Determine the weight of packet queue in the traffic class based on WFQ.

Step 2: Estimate the traversed hop count of selected packet in traffic class.

Step 3: THCT maintains the packet queues which is sorted based on traversed hop count

Step 4: THCT implement a search operation with worst and average case complexity $\mathrm{O}(\log n)$ in order to search a new packet and a shift operation method to make free space before a new insertion of packet.

Step 5: In the node set, $S$ steals the time and transmit the packet if packet is queued for longer time and there is no response from the primary node

Hence, the proposed technique helps to make efficient utilization of the channel without any delay in the network.

The multi-layer node is first classified into the primary sender node and the secondary sender node. After that based on the THCT and WFQ is allowed for the transmission. In case the primary sender node is in silent and timeslot is longer secondary sender node steals the time and used for transmission.

\section{Simulation Results}

\subsection{Simulation Model and Parameters}

Table 1. Simulation settings and parameters

\begin{tabular}{|c|c|}
\hline No. of Nodes & 100 \\
\hline Area Size & 900 X 900 \\
\hline Mac & IEEE 802.11 \\
\hline Transmission Range & $250 \mathrm{~m}$ \\
\hline Simulation Time & 50 sec \\
\hline Traffic Source & CBR and TCP \\
\hline Packet Size & 512 \\
\hline Flows & $2,4,6,8$ and 10 \\
\hline Rate & $\begin{array}{c}100,200,300,400 \\
\text { and } 500 \mathrm{~Kb}\end{array}$ \\
\hline
\end{tabular}

The Network Simulator (NS2) [19], is used to simulate the proposed architecture. In the simulation, 50 mobile nodes move in a 900 meter x 900 meter region for 50 seconds of simulation time. All nodes have the same transmission range of 250 meters. The simulated traffic is Constant Bit Rate (CBR) and TCP.

\subsection{Performance Metrics}

The proposed Delay Sensitive and Interference Aware MAC Protocol (DSIAMAC) is compared with the multi-layer MAC (MLMAC) protocol [18]. The performance is evaluated mainly, according to the following metrics.

- Packet Delivery Ratio: It is the ratio between the number of packets received and the number of packets sent.

- Packet Drop: It refers the average number of packets dropped during the transmission

- Energy Consumption: It is the amount of energy consumed by the nodes to transmit the data packets to the receiver.

- Delay: It is the amount of time taken by the nodes to transmit the data packets. 


\subsection{Results}

\section{Case-1 (CBR scenario)}

\section{1) Based on Flows}

In our first experiment we vary the number of flows as $2,4,6,8$ and 10 .

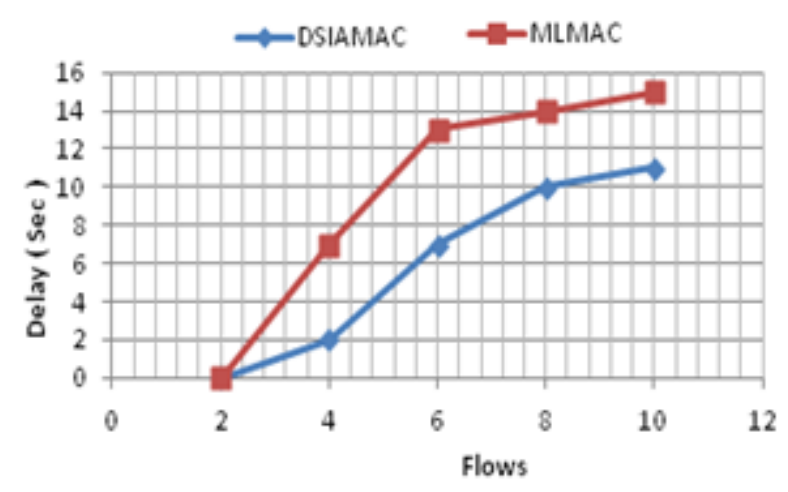

Figure.4 Flows Vs Delay

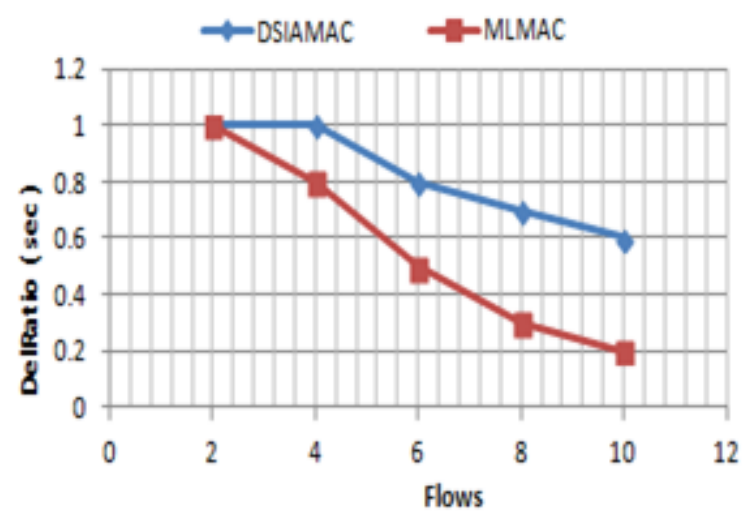

Figure.5 Flows Vs Delivery Ratio

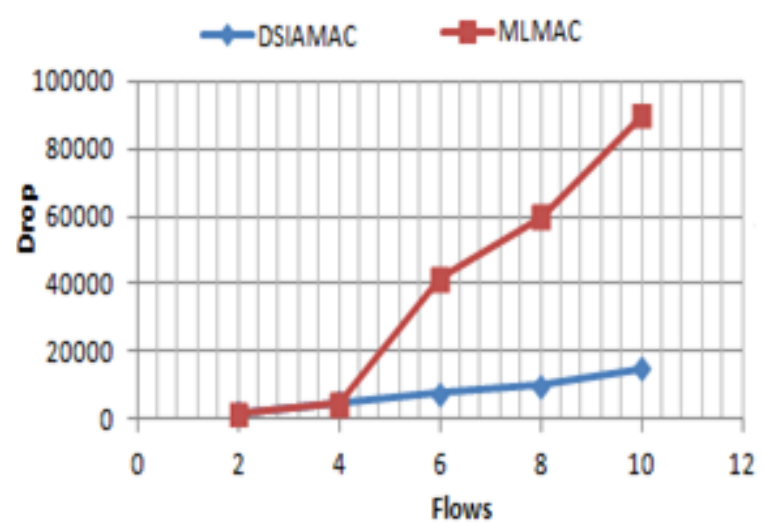

Figure.6 Flows Vs Drop

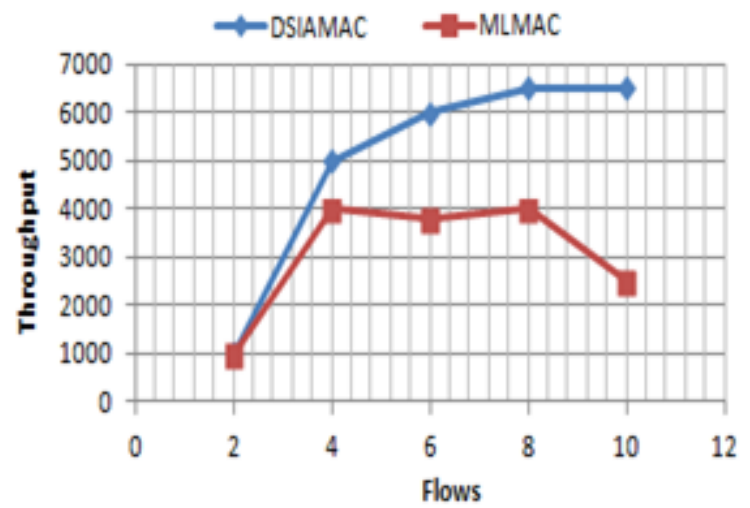

Figure.7 Flows Vs Throughput

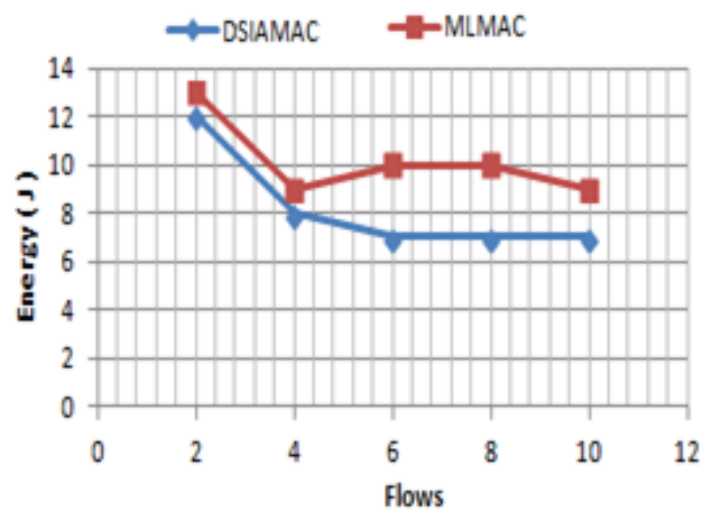

Figure.8 Flows Vs Energy Consumption

Figure 4 shows the delay of DSIAMAC and MLMAC techniques for different number of flows scenario. We can conclude that the delay of our proposed DSIAMAC approach has $50 \%$ of less than MLMAC approach.

Figure 5 shows the delivery ratio of DSIAMAC and MLMAC techniques for different number of flows scenario. We can conclude that the delivery ratio of our proposed DSIAMAC approach has $29 \%$ of higher than MLMAC approach.

Figure 6 shows the drop of DSIAMAC and MLMAC techniques for different number of flows scenario. We can conclude that the drop of our proposed DSIAMAC approach has 54\% of less than MLMAC approach.

Figure 7 shows the throughput of DSIAMAC and MLMAC techniques for different number of flows scenario. We can conclude that the throughput of our proposed DSIAMAC approach has $29 \%$ of higher than MLMAC approach.

Figure 8 shows the energy consumption of DSIAMAC and MLMAC techniques for different number of flows scenario. We can conclude that the energy consumption of our proposed DSIAMAC approach has $22 \%$ of less than MLMAC approach. 


\section{Case-2 (TCP scenario)}

\section{1) Based on Flows}

In this experiment we vary the number of flows as $2,4,6,8$ and 10 .

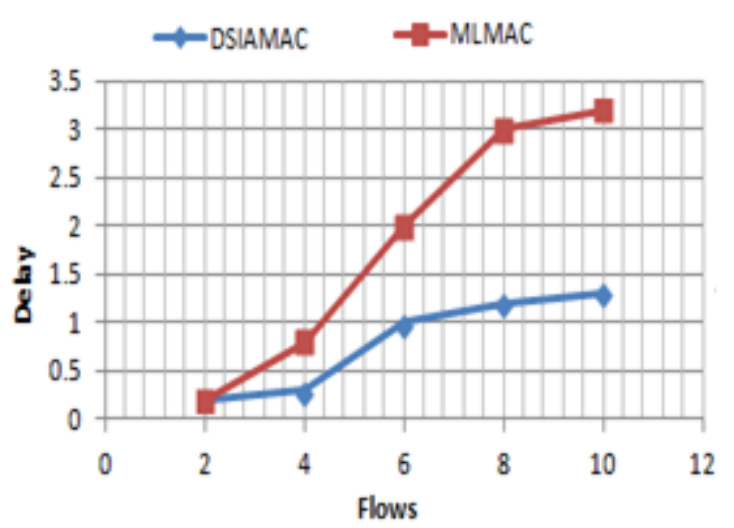

Figure.9 Flows Vs Delay

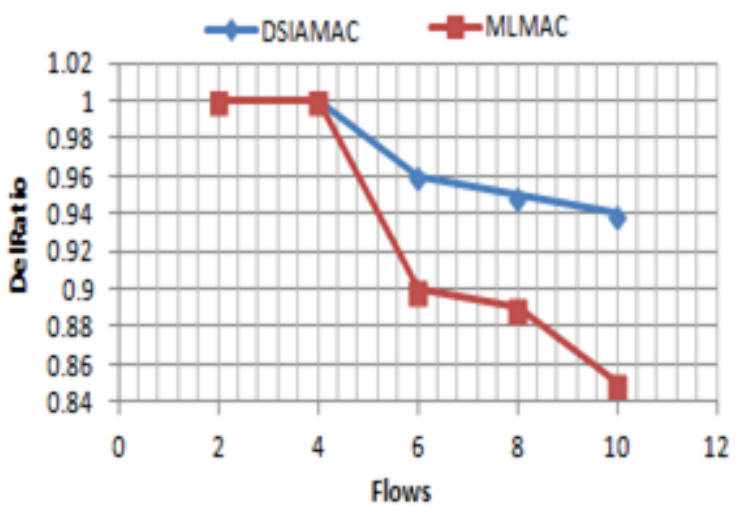

Figure.10 Flows Vs Delivery Ratio

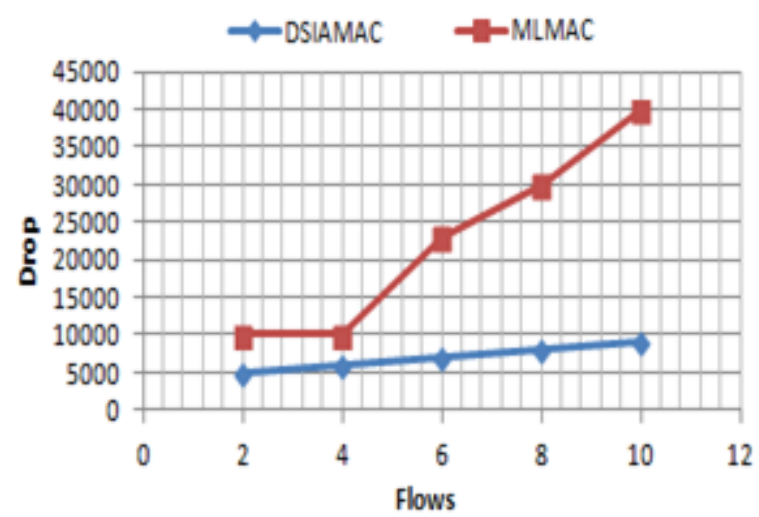

Figure.11 Flows Vs Drop

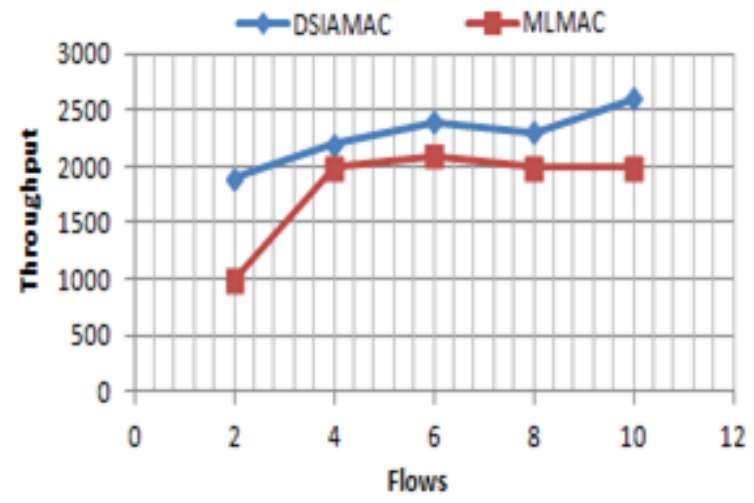

Figure.12 Flows Vs Throughput

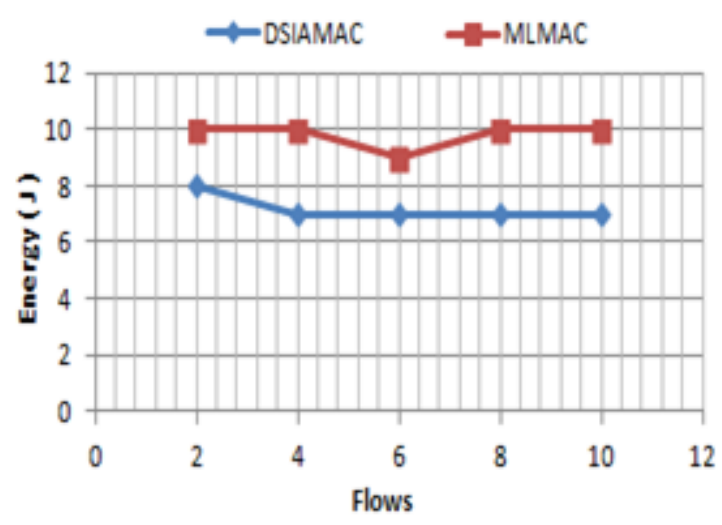

Figure.13 Flows Vs Energy Consumption

Figure 9 shows the delay of DSIAMAC and MLMAC techniques for different number of flows scenario. We can conclude that the delay of our proposed DSIAMAC approach has $40 \%$ of less than MLMAC approach.

Figure 10 shows the delivery ratio of DSIAMAC and MLMAC techniques for different number of flows scenario. We can conclude that the delivery ratio of our proposed DSIAMAC approach has $4 \%$ of higher than MLMAC approach.

Figure 11 shows the drop of DSIAMAC and MLMAC techniques for different number of flows scenario. We can conclude that the drop of our proposed DSIAMAC approach has $62 \%$ of less than MLMAC approach.

Figure 12 shows the throughput of DSIAMAC and MLMAC techniques for different number of flows scenario. We can conclude that the throughput of our proposed DSIAMAC approach has $26 \%$ of higher than MLMAC approach.

Figure 13 shows the energy consumption of DSIAMAC and MLMAC techniques for different number of flows scenario. We can conclude that the energy consumption of our proposed DSIAMAC approach has 19\% of less than MLMAC approach. 


\section{2) Based on Rate}

In this experiment we vary the transmission rate as $100,200,300,400$ and $500 \mathrm{~Kb}$.

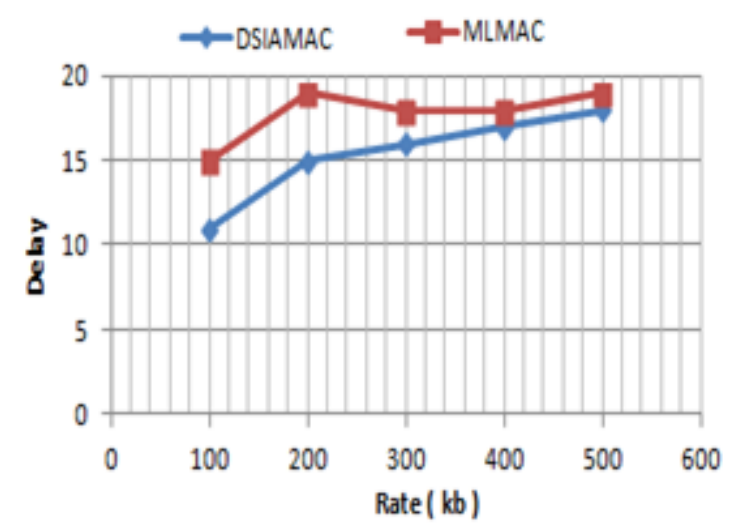

Figure.14 Rate Vs Delay

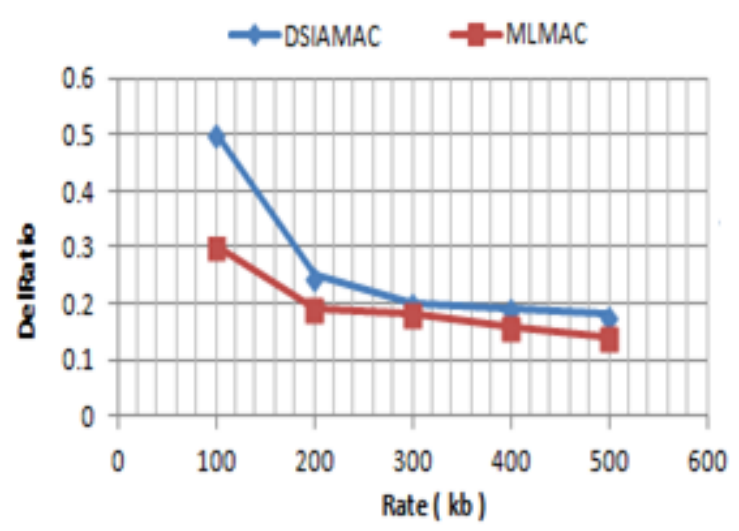

Figure.15 Rate Vs Delivery Ratio

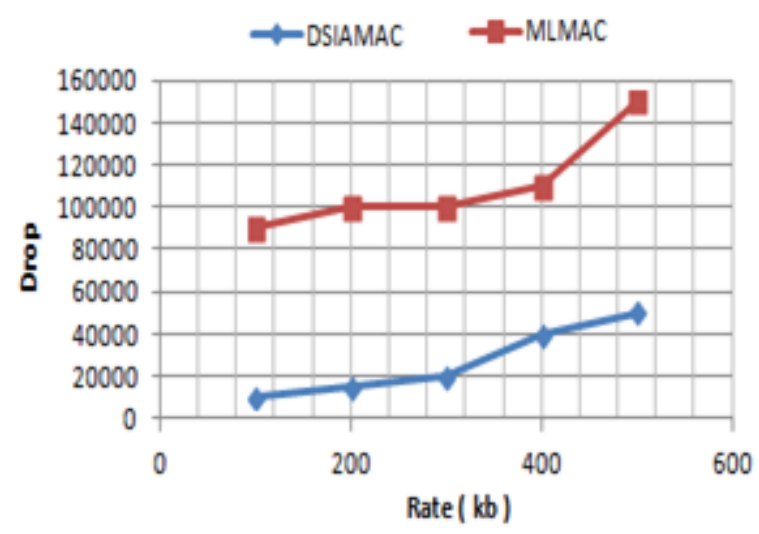

Figure.16 Rate Vs Drop

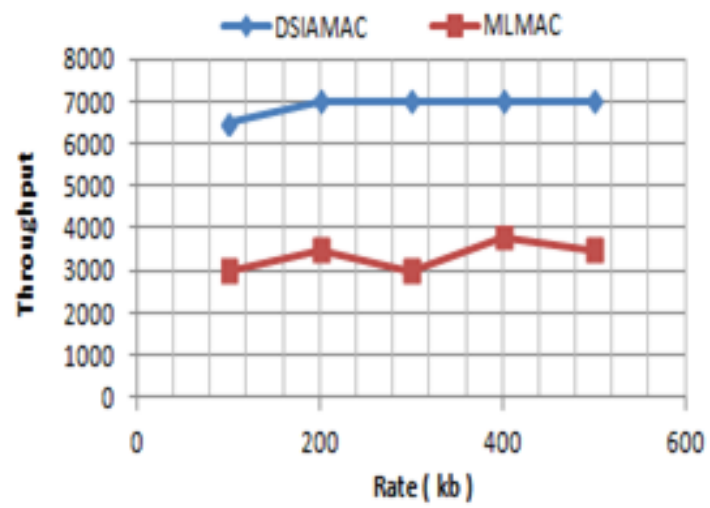

Figure.17 Rate Vs Throughput

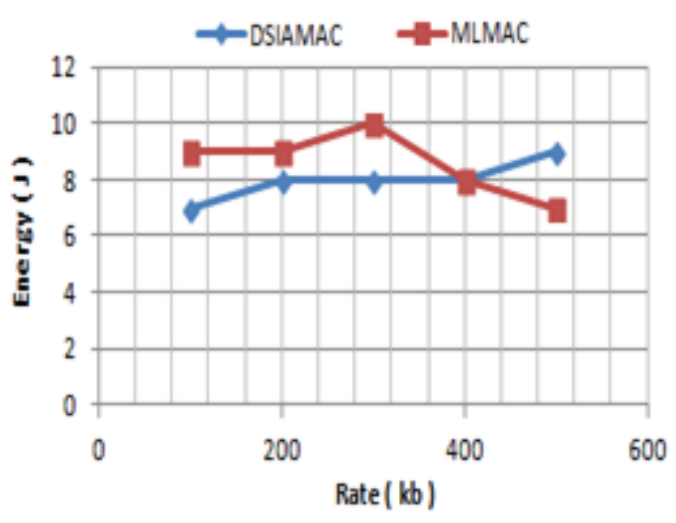

Figure.18 Rate Vs Energy Consumption

Figure 14 shows the delay of DSIAMAC and MLMAC techniques for different rate scenario. We can conclude that the delay of our proposed DSIAMAC approach has $14 \%$ of less than MLMAC approach.

Figure 15 shows the delivery ratio of DSIAMAC and MLMAC techniques for different rate scenario. We can conclude that the delivery ratio of our proposed DSIAMAC approach has 50\% of higher than MLMAC approach.

Figure 16 shows the drop of DSIAMAC and MLMAC techniques for different rate scenario. We can conclude that the drop of our proposed DSIAMAC approach has $72 \%$ of less than MLMAC approach.

Figure 17 shows the throughput of DSIAMAC and MLMAC techniques for different rate scenario. We can conclude that the throughput of our proposed DSIAMAC approach has 50\% of higher than MLMAC approach.

Figure 18 shows the energy consumption of DSIAMAC and MLMAC techniques for different rate scenario. We can conclude that the energy consumption of our proposed DSIAMAC approach has $19 \%$ of less than MLMAC approach. 


\section{Conclusion}

In this paper a delay sensitive and interference aware MAC protocol for WSN is proposed. First MAC protocol is proposed which divides network into layer and assign node to each layer to avoid data loss in the network. Once network is divided to the multi-layer, timeslot is assigned to each node. To utilize the channel efficiently, timeslot steal mechanism is implemented. In this mechanism, the nodes will listen to channel carefully and if the primary sender node is in idle, then the secondary node utilize that time and transmits the packet. To avoid interference, a hybrid prioritization scheme is implemented to prioritize the packet queue based on weight of the packet queue and based on the idle time of the prioritized packet. As a future work we can add adaptive time slot mechanism that will result in improving the delay and hence the system will perform better.

\section{References}

[1] M. I. Channa and I. Memon, "Real Time Traffic Support in Wireless Sensor Networks", Student Conference on Engineering Sciences and Technology, SCONEST , pp. 1-6, 2005.

[2] T. R. Sheltami and E. Shakshuki, "Neighbor-Aware Cluster head with Different Sleep Scheduling Protocols", International Conference on Parallel Processing - Workshops, pp. 143-147, 2008.

[3] N. Bandirmali, I. Erturk and C. Ceken, "Securing Data Transfer in Delay-sensitive and Energy-aware WSNs Using the Scalable Encryption Algorithm", 4th International Symposium on Wireless Pervasive Computing, ISWPC 2009.

[4] B. M. Khan and F. H. Ali, "Mobility Adaptive Energy Efficient and Low Latency MAC for Wireless Sensor Networks", Fifth International Conference on Next Generation Mobile Applications and Services, pp. 218-223, 2011.

[5] S. A. Hameed, E. M. Shaaban, H. M. Faheem and M. S. Ghoniemy, "Mobility-Aware MAC Protocol for Delay-Sensitive Wireless Sensor Networks", International Conference on Ultra Modern Telecommunications \& Workshops, ICUMT, pp. 1-8, 2009.

[6] S. Gherairi, S. Ouni, and F. Kamoun, "Optimized TDMA Multi-Frequency Scheduling Access Protocols for Sensor Networks", International Conference on Communications, Computing and Control Applications (CCCA), pp. 1-6, 2011.

[7] H. R. Oh and H. Song, "An Energy-aware Wireless Sensor MAC Protocol for Delay-Sensitive Data Transmission", 5th International Symposium on
Wireless Pervasive Computing (ISWPC), pp. 69-73, 2010.

[8] F. D. Rango, F. Veltri, D. Critelli, P. Fazio and S. Marano, "Interference-Aware Ad-hoc on Demand Distance Vector (IA-AODV) Protocol", International Symposium on Performance Evaluation of Computer \& Telecommunication Systems, Vol.41, pp. 170-177, 2009.

[9] F. D. Rango, F. Veltri, D. Critelli, P. Fazio and S. Giacco, "An Interference Aware Approach for Routing in UWB Networks", IEEE 70th Vehicular Technology Conference Fall (VTC), 2009.

[10] H. Huang, G. Hu, F. Yu and Z. Zhang, "Energyaware interference-sensitive geographic routing in wireless sensor networks", IET Communications, Vol. 5, No. 18, 2011.

[11] M. Doudou, D. Djenouri, and N. Badache, "Survey on Latency Issues of Asynchronous MAC Protocols in Delay-Sensitive Wireless Sensor Networks", IEEE Communications Surveys \& Tutorials, Vol. 15, No. 2, pp. 528-550, 2013.

[12] D. D. Chaudhary and Dr. L. M. Waghmare, "Quality of Service Analysis in Wireless Sensor Network by Controlling End-to-End Delay", 7th IEEE Conference on Industrial Electronics and Applications (ICIEA), pp. 703-708, 2012.

[13] M. A. Yigitel, O. D. Incel and C.Ersoy, "Design and implementation of a QoS-aware MAC protocol for Wireless Multimedia Sensor Networks", Computer Communications, Vol. 34, No. 16, pp. 1991-2001, 2011.

[14] M. A. Hamid, M. A. A. Wadud and I. Chong, "A Schedule-based Multi-channel MAC Protocol for Wireless Sensor Networks", Sensors, Vol. 10, No. 10, pp. 9466-9480, 2010.

[15] M. H. S. Gilani, I. Sarrafi and M. Abbaspour, "An adaptive CSMA/TDMA hybrid MAC for energy and throughput improvement of wireless sensor networks", Ad Hoc Networks, Vol. 44, No. 4, pp. 1297-1304, 2011.

[16] O. D. Incel, L. Hoesel, P. Jansen and P. Havinga, "MC-LMAC: A multi-channel MAC protocol for wireless sensor networks", Ad Hoc Networks, Vol. 9, No. 1, pp. 73-94, 2011.

[17] W. L. Tan, W. C. Lau and O. C. Yue, "Performance analysis of an adaptive, energy-efficient MAC protocol for wireless sensor networks", Journal of Parallel Distribution and Computing, Vol. 72, No. 4, pp. 504-514, 2012.

[18] R. Thalore, J. Sharma, M. Khurana and M. K. Jha, "QoS evaluation of energy-efficient ML-MAC protocol for wireless sensor networks", International Journal of Electronics and Communications, Vol. 67, No. 12, pp. 1048-1053, 2013.

[19] Network Simulator: http:///www.isi.edu/nsnam/ns 